Imputation for missing values confirmed these results. Hypoglycemia evoked by TGC did not negatively affect neurocognitive outcome.

Conclusion Despite hypoglycemia, TGC in PICU did not harm neurocognitive development 4 years later.

\section{CARDIAC FUNCTION AT THE AGE OF 7 YEARS OF REGIONAL BIRTH COHORT OF EXTREMELY LOW BIRTH WEIGHT INFANTS $(<$ 1000G)}

doi:10.1136/archdischild-2012-302724.0151

P Kwinta, M Jagla, A Grudzien, M Klimek, M Zasada, JJ Pietrzyk. Department of Pediatrics, Jagiellonian University, Cracow, Poland

Aim Assessment of long-term cardiac complications in the regional cohort of extremely low birth weight (ELBW) children born in 2002-2004.

Material and Methods The study group comprising 81 children born as ELBW infants with the median birthweight of $890 \mathrm{~g}\left(25^{\text {th }}\right.$ $75^{\text {th }}$ percentile: $760-950$ ) were evaluated at the mean age of 7 years. The control group included 40 children born full term. Echocardiography and 24-hour ambulatory blood pressure measurements were performed.

Results

Abstract 151 Table $1 \quad$ M-mode variables (presented as z-score)

\begin{tabular}{llll}
\hline & ELBW & Control & p \\
\hline RVDd & $-1.49 \pm 1,25$ & $-0.31 \pm 0,91$ & $<0.001$ \\
LVIDd & $-0.53 \pm 1,26$ & $0.13 \pm 0,94$ & 0.01 \\
Ao & $0.24 \pm 1,23$ & $0.83 \pm 1,03$ & 0.02 \\
LA & $-0.93 \pm 1,07$ & $-0.15 \pm 1,02$ & $<0.01$ \\
\hline
\end{tabular}

Abstract 151 Table 2 Results of 24-hour blood pressure monitoring

\begin{tabular}{llll}
\hline & ELBW & Control & p \\
\hline 24-h mean MAP(mmHg) & $79 \pm 5.9$ & $77 \pm 4.4$ & 0.2 \\
Systolic BP load(\%) & $28 \pm 22$ & $16 \pm 14$ & $<0.01$ \\
Diastolic BP load(\%) & $27 \pm 20$ & $17 \pm 10$ & $<0.01$ \\
Mean HR(bpm) & $93 \pm 8$ & $87 \pm 7$ & $<0.001$ \\
\hline
\end{tabular}

Abstract 151 Table 3 LVM, LVMI and cardiac index

\begin{tabular}{llll}
\hline & ELBW & Control & p \\
\hline LVM(g) & $48.8 \pm 15,5$ & $65.1 \pm 15,4$ & $<0.001$ \\
LVMI(g/m2.7) & $32.4 \pm 9,2$ & $35.7 \pm 8,3$ & 0.08 \\
Stroke index(/BSA) & $41.8 \pm 15,3$ & $47.9 \pm 12,5$ & 0,04 \\
Cl (I/m2) & $3.6 \pm 1,2$ & $3.8 \pm 1,04$ & 0.49 \\
\hline
\end{tabular}

Conclusions The former ELBW children have smaller heart's diameters and to reach the same cardiac index their heart rate is faster. Moreover, the former ELBW children have higher blood pressure comparing to their peers.

\section{PREDICTIVE FACTORS FOR SURVIVAL AFTER PAEDIATRIC OUT-OF-HOSPITAL CARDIAC ARREST: A UK MULTICENTRE COHORT STUDY}

doi:10.1136/archdischild-2012-302724.0152

1,2B Scholefield, ${ }^{3} \mathrm{~S}$ Raman, ${ }^{3} \mathrm{~A}$ Hussey, ${ }^{4} \mathrm{~F}$ Haigh, ${ }^{1} \mathrm{HK}$ Kanthimathinathan, ${ }^{3} \mathrm{~S}$ Skellett, ${ }^{3} \mathrm{M}$ Peters, 'H Duncan, 'K Morris. 'Paediatric Intensive Care Unit, Birmingham Children's Hospital, Birmingham; ${ }^{2}$ Medical School, University of Warwick, Warwick; ${ }^{3}$ Paediatric Intensive Care Unit, Great Ormond Street Hospital, London; ${ }^{4}$ Paediatric Intensive Care Unit, Alder Hey Childrens' Hospital, Liverpool, UK
Introduction Survival after paediatric out-of-hospital cardiac arrest (OHCA) is poor, even amongst those who are successfully resuscitated and admitted to PICU. Better prediction of survival would be of benefit to clinical teams and to research teams designing trials. This study aimed to identify predictive factors for survival to inform the design of a post-OHCA intervention trial.

Method Retrospective, cohort study of 155 infants and children ( 1 day to 16 years) admitted to 3 UK PICUs after OHCA (20042010). Variables relating to a) the resuscitation period (Utstein) and b) the post-resuscitation period were included in two multivariate stepwise regression models to identify predictive factors for survival to PICU discharge.

Results $32 \%$ (50/155) children survived to PICU discharge. Resuscitation variables individually associated with improved survival included; presenting in a shockable rhythm, shorter duration of arrest, return of spontaneous cardiac output prior to arrival in the emergency department and lower number of epinephrine doses. Post-resuscitation variables individually associated with improved survival included; higher arterial $\mathrm{pH}$, lower blood lactate, lower maximum glucose, higher base excess and responsive pupils. Results of multivariate stepwise regression models are reported in table.

Conclusion This large UK study is the first to identify lactate as one of the key predictors of paediatric OHCA survival in patients admitted to PICU. Development of an accurate prediction tool would assist trial design and prognostication after paediatric OHCA.

\begin{tabular}{|c|c|c|c|}
\hline Variable & $\begin{array}{l}\text { Odds Ratio } \\
\text { for survival }\end{array}$ & $95 \% \mathrm{Cl}$ & $P$ value \\
\hline \multicolumn{4}{|l|}{ Model 1: Resuscitation factors } \\
\hline $\begin{array}{l}\text { No epinephrine (versus one or more } \\
\text { doses of epinephrine during resuscitation) }\end{array}$ & 11.98 & 2.31 .61 .99 & 0.003 \\
\hline VFNT (versus PEAlasystolebradycardia) & 3.95 & $0.96-16.27$ & 0.057 \\
\hline \multicolumn{4}{|c|}{ (Model 1 : Area under receiver operating cunve $=0.67$ ) } \\
\hline \multicolumn{4}{|l|}{ Model 2: Post-resuscitation factors } \\
\hline Two unresponsive pupils (versus reactive) & 0,19 & $0.07-0.52$ & 0.001 \\
\hline Blood Lactate (per 1.0 mmoll increment) & 0.85 & $0.75-0.87$ & 0.015 \\
\hline \multicolumn{4}{|c|}{ Q Model 2: Ares under receiver operating curve $=0.83$ ) } \\
\hline
\end{tabular}

\begin{tabular}{l}
\hline 153 VISUOSPATIAL AND EXECUTIVE FUNCTION IN \\
ADOLESCENT PATIENTS WITH CONGENITAL HEART \\
DISEASE
\end{tabular}

doi:10.1136/archdischild-2012-302724.0153

1,2M von Rhein, ${ }^{2} \mathrm{M}$ Kugler, ${ }^{2 B}$ Latal. 'Developmental Pediatrics, Kantonsspital, Winterthur; ${ }^{2}$ Child Development Center, University Children's Hospital, Zurich, Switzerland

Background and aims Visuospatial deficits have been described for 8 year-old patients with congenital heart disease (CHD) after bypass surgery based on the performance in the Rey-Osterrieth complex figure test (ROCFT). It is unknown whether these deficits persist into adolescence and which scoring systems is optimal to score performance in the ROCFT. We therefore performed ROCFT in adolescent CHD patients and healthy controls, and compared performance according to three different scoring methods.

Methods We examined 53 adolescents (mean age 13.7 years, 44\% male, $50 \%$ cyanotic CHD) with $\mathrm{CHD}$ after open-heart surgery 
(mean age at surgery: 1.4 years) and 32 healthy subjects. ROCFT was scored according to three different validated scoring methods.

Results Results varied markedly between the scoring methods. When scored according to Meyers \& Meyers, patients performed significantly worse than controls in the copy task ( $\mathrm{p}=0.03$ ), whereas no significant differences were found in the memory task. Scoring according to Wallon and Mesmin showed clear differences between subjects and controls with respect to the approach how to construct the figure: $78 \%$ of CHD patients (controls: $47 \%$ ) displayed a unstructured or intermediate approach to drawing the figure, whereas only $22 \%$ of CHD patients (controls: $53 \%$ ) chose a complex approach $(\mathrm{p}=0.001)$. Scoring according to Bernstein et al. showed no differences between groups.

Conclusions Adolescents with surgically treated CHD demonstrate deficits in visuospatial and executive function. The ROCFT provides information on different functional aspects, which cannot adequately be assessed with one single scoring method.

\section{BORN TOO SMALL OR TOO EARLY - THE EFFECT OF PHYSICAL FITNESS IN YOUNG ADULTHOOD ON COGNITIVE PERFORMANCE}

doi:10.1136/archdischild-2012-302724.0154

J Svedenkrans, E Henckel, J Kowalski, K Bohlin, M Norman. Department of Clinical Science, Intervention and Technology, Karolinska Institute, Stockholm, Sweden

Background and aims Physical exercise has been demonstrated to give positive cognitive effects. We have previously reported lower exercise capacity in otherwise healthy young men with low birth weight or preterm birth. The objective of this study was to investigate the association between perinatal risk factors, cognitive performance and physical fitness in young adulthood.

Methods This was a population-based cohort study including 218,915 young men born in Sweden 1973-1981, conscripted for military service in 1993-2001. Data on birth characteristics was obtained from the Medical Birth Register and linked to information on cognitive test scores and results on ergometer cycling test from the Military Conscript Register.

Results Exercise capacity, as measured by ergometer cycling, was positively associated with cognitive performance, as measured by global intelligence stanine scores, $p<0.001$. The subgroup with shortest gestational age, less than 28 weeks, exhibited both the lowest cognitive test scores and the lowest exercise capacity. Low birth weight standard deviation scores (BWSDS) for gestational age was associated with lower cognitive performance in a stepwise manner. Using ordinal regression analysis, low BWSDS remained independently associated with low cognitive scores in young adult age.

Conclusions The lower cognitive performance observed in young adults born prematurely or with low birth weight is associated with physical fitness. Targeting physical exercise may be an intervention to enhance cognitive performance and educational achievements in a population at risk, such as survivors of preterm birth and poor fetal growth.

\section{EFFECT OF IN UTERO EXPOSURE TO ISCHEMIC-HYPOXIC CONDITIONS ON CHILDHOOD ATTENTION DEFICIT HYPERACTIVITY DISORDER}

doi:10.1136/archdischild-2012-302724.0155

${ }^{1,2} \mathrm{D}$ Getahun, ${ }^{3} \mathrm{GG}$ Rhoads, ${ }^{3} \mathrm{~K}$ Demissie, ${ }^{4} \mathrm{~L}$ Shou-En, 'VP Quinn, ${ }^{5} \mathrm{MJ}$ Fassett, ${ }^{6} \mathrm{DA}$ Wing, 'SJ Jacobsen. 'Research \& Evaluation, Kaiser Permanente Southern California Medical Group, Pasadena, CA; ${ }^{2}$ Obstetrics \& Gynecology, UMDNJ - Robert Wood Johnson Medical School, New Brunswick; ${ }^{3}$ Epidemiology; ${ }^{4}$ Biostatistics, UMDNJ - School of Public Health, Piscataway, NJ; ${ }^{5}$ Maternal-Fetal Medicine, Kaiser Permanente Southern California Medical Group, Pasadena; ${ }^{6}$ Obstetrics \& Gynecology, University of California, Irvine, Irvine, CA, USA
Objective To examine the association between ischemic-hypoxic conditions (IHC) and Attention Deficit Hyperactivity Disorder (ADHD) by gestational age at delivery and race/ethnicity.

Methods A nested case-control study using the Kaiser Permanente Southern California (KPSC) medical records. Study cohort were children aged 5-11 years who were delivered and cared for in KPSC Healthcare system between 1995-2010 ( $\mathrm{n}=308,634$ ). Cases were children with a clinical diagnosis of $\mathrm{ADHD}$ and obtained at least 2 prescriptions specific to ADHD during the follow-up period. For each case, five controls matched to cases on child age at time of diagnosis were selected. Exposures were defined based on ICD-9 codes. A conditional logistic regression model was used to estimate adjusted odds ratios (OR).

Results Among eligible children, 13,613 (4.3\%) had a diagnosis of ADHD. Compared to control children, case children were more likely to be male and of White or African-American race/ethnicity. Case children than controls were more likely to be exposed to IHC $(\mathrm{OR}=1.16,95 \%$ confidence intervals [CI] 1.11-1.21). Analysis of cases and controls stratified by gestational age revealed that case children born at 28-33, 34-36, and 37-42 weeks of gestation, were significantly more likely to be exposed to IHC; 1.6 -fold $(95 \%$ CI, 1.2-2.2), 1.2-fold (95\% CI, 1.0-1.4), and 1.1-fold (95\% CI, 1.0-1.2), respectively, compared to control children. IHC was associated with increased odds of $\mathrm{ADHD}$ across all race/ethnicity groups.

Conclusion These findings suggest that IHC is independently associated with an increased risk of childhood $\mathrm{ADHD}$ especially in early preterm birth.

\section{CEFAZOLIN PLASMA PROTEIN BINDING AND ITS COVARIATES IN NEONATES}

doi:10.1136/archdischild-2012-302724.0156

${ }^{1} \mathrm{~A}$ Smits, ${ }^{2,3} \mathrm{~A}$ Kulo, ${ }^{2} \mathrm{R}$ Verbesselt, ${ }^{1} \mathrm{~V}$ Cossey, ${ }^{2} \mathrm{~J}$ de Hoon, ${ }^{4} \mathrm{P}$ Vermeersch, ${ }^{1} \mathrm{~K}$ Allegaert. ${ }^{1}$ Neonatal Intensive Care Unit; ${ }^{2}$ Center for Clinical Pharmacolgoy, University Hospitals

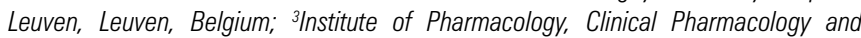
Toxicology, Faculty of Medicine, University of Sarajevo, Sarajevo, Bosnia-Herzegovina; ${ }^{4}$ Department of Laboratory Medicine, University Hospitals Leuven, Leuven, Belgium

Background and aim Cefazolin (CFZ) is highly and saturably bound to albumin in adults. It is mainly used as prophylactic antibiotic agent. The aim of the present study is to describe CFZ protein binding and its covariates in neonates.

Methods Neonates to whom intravenous CFZ (50 mg/kg) was administered as standard care prior to an invasive procedure were included. Total and unbound CFZ plasma concentrations were determined at $1 / 2 ; 2 ; 4$ and 8 hours after CFZ administration. Linear and multiple regression analyses were used to document covariates of unbound CFZ fraction. For paired analysis of unbound CFZ fractions Wilcoxon signed rank test was used.

Results Forty patients with median weight 2767 (range 830-4200) grams and median postmenstrual age (PMA) 39 (25-45) weeks were included. Median unbound CFZ fraction was 0.39 (0.10-0.73). Linear regression of unbound CFZ fraction versus unbound CFZ plasma concentration $\left(R^{2}=0.39\right)$ had a slope significantly different from zero $(p<0.001)$. In a multiple regression analysis, albuminaemia, total CFZ concentration, indirect bilirubinaemia and PMA resulted in an $\mathrm{R}^{2}$ value of 0.496 . Median unbound CFZ fraction at peak concentration $(0.46$; range $0.28-0.69)$ was significantly higher compared to trough level (0.36; range 0.17-0.73) $(p<0.001)$.

Conclusions Between patient and within patient saturability of CFZ protein binding were also documented in neonates. We revealed a median plasma unbound CFZ fraction of 0.39 in neonates, which is higher than reported values in adults. The integration of CFZ protein binding aspects in future pharmacokinetic/pharmacodynamic research is warranted to optimize CFZ dosing, especially in neonates. 